\title{
A Numerical Study of Mixed Convection in Square Lid-Driven with Internal Elliptic Body and Constant Flux Heat Source on the Bottom Wall
}

\author{
M. J. H. Munshi ${ }^{1 *}$ M. A. Alim ${ }^{2}$, M. Ali ${ }^{3}$, M. S. Alam ${ }^{4}$ \\ ${ }^{1}$ Department of Mathematics, Hamdard University Bangladesh (HUB), Hamdard Nagar, \\ Gazaria, Munshigonj- 1510, Bangladesh \\ ${ }^{2}$ Department of Mathematics, Bangladesh University of Engineering and Technology \\ (BUET), Dhaka- 1000, Bangladesh \\ ${ }^{3}$ Department of Mathematics, Chittagong University of Engineering and Technology \\ (CUET), Chittagong- 4349, Bangladesh \\ ${ }^{4}$ Department of Mathematics, Dhaka University of Engineering and Technology \\ (DUET), Gazipur- 1700, Bangladesh
}

Received 17 September 2016, accepted in final revised form 11 January 2017

\begin{abstract}
The mixed convection in square lid-driven with internal elliptic body and constant flux heat source on the bottom wall is numerically simulated in this paper following a finite element method approach. The left moving wall and right moving wall are cold. The upper wall is insulated and so is the lower wall with heat flux located in the middle. The magnetic field of strength $B$ is applied parallel to $\mathrm{x}$ - axis. Result is presented for different Richardson numbers $(0.01 \leq \mathrm{Ri} \leq 10)$ when Grashof numbers are $(10 \leq \mathrm{Gr} \leq$ 50) and Prandtl number is taken as $P r=0.733$ for all computations. The influence of the Richardson number on heat source surface is being investigated in this paper. Results are presented in the form of streamline and isotherm plots as well as the variation of the maximum temperature and Nusselt number at the heat source surface under different conditions. A detailed analysis of flow pattern shows that the mixed convection is based on the parameters Richardson number $(R i)$, Grashof number $(G r)$ and Reynolds number $(R e)$.
\end{abstract}

Keywords: Mixed convection; Square cavity; Elliptic obstacle; Lid-driven cavity and FEM.

(C) 2017 JSR Publications. ISSN: 2070-0237 (Print); 2070-0245 (Online). All rights reserved. doi: http://dx.doi.org/10.3329/jsr.v9i2.29644 J. Sci. Res. 9 (2), 145-158 (2017)

\section{Introduction}

Combined forced and free convection flows, or mixed convection flows, arise in many transport processes in engineering devices in nature. Examples of mixed convection

* Corresponding author: jahir.buet.bd@gmail.com 
processes can be found in connection with heat exchangers, hot-wise anemometers, nuclear reactors, electronic devices, atmospheric boundary-layer flows, solar collectors, energy storage and heat rejection systems, etc. [1]. Al-Amiri et al. [2] investigated numerically steady mixed convection in a square lid-driven cavity under the combined buoyancy effects of thermal and mass diffusion. Sharif [3] studied numerically laminar mixed convective heat transfer in two-dimensional shallow rectangular driven cavities of aspect ratio 10. The top moving lid of the cavity is at a higher temperature than the bottom wall. The effects of inclination of the cavity on the flow and thermal fields are investigated. The stream line and isotherm plots and the variation of the local and average Nusselt numbers at the hot and cold walls are presented. Khanafer et al. [4] investigated numerically unsteady laminar mixed convection heat transfer in a lid driven cavity. In addition, the two vertical walls of the enclosure are kept insulated. Fluid flow and heat transfer characteristics are examined in the domain of the Reynolds number, Grashof number and the dimensionless lid oscillation frequency. Rahman et al. [5] investigated numerically the conjugate effect of joule heating and magnetic force, acting normal to the left vertical wall of an obstructed lid-driven cavity saturated with an electrically conducting fluid. Saha et al. [6] have performed the numerical simulation of the mixed convection flow and heat transfer in a lid-driven cavity with wavy bottom surface. They observed that the heat transfer mechanisms and flow characteristics inside the cavity noticeably depend on the number of undulations, Grashof number and Reynolds number. Rahman et al. [7] analyzed mixed convection in a rectangular cavity with a heat conducting horizontal circular cylinder by using finite element method. Saha et al. [8] have performed the numerical effect of internal heat generation or absorption on magnetohydrodynamic (MHD) mixed convection flow in a lid driven cavity. They significant reduction in the average Nusselt number was produced as the strength of the applied magnetic field increased. In addition, heat generation predicated to decrease the average Nusselt number whereas heat absorption increases it. Saha et al. [9] numerically investigated the hydro-magnetic mixed convection flow in a lid driven cavity with wavy bottom surface. They observed that the wavy lid-driven cavity can be considered as an effective heat transfer mechanism in presence of magnetic field at large wavy surface amplitude and low Richardson numbers. Guanghong et al. [10] have investigated the mixed convection in rectangular cavities at various aspect ratios with moving isothermal sidewalls and constant flux heat source on the bottom wall. Hussein [11] investigated the mixed convection in square lid-driven with Eccentric Circular Body. Munshi et al. [12] analyzed a numerical study of mixed convection in square liddriven with internal elliptic body and constant flux heat source on the bottom wall. Nasrin [13] carried out a aspect ratio effect of vertical lid-driven chamber having a centered conducting solid on mixed magneto convection. Hussain et al. [14] studied the problem of mixed convection heat transfer in a differentially heated square enclosure with a conductive rotating circular cylinder at different vertical locations. Sivasankaran et al. [15] considered the numerical study on mixed convection in an 
inclined lid-driven cavity with discrete heating. Sivasankaran et al. [16] investigated mixed convection in a lid-driven two-dimensional square cavity with corner heating and internal heat generation. Hussein [17] paper gives a comprehensive over view and understanding related with mixed convection in cylinders. Hussein et al. [18] measured the characteristics of MHD mixed convection in a parallel motion two-sided lid-driven differentially heated parallelogrammic cavity with various skew angles. Saha et al. [19] rigorously investigated mixed convection in a tilted lid-driven square enclosure with adiabatic cylinder at the center. Hussein et al. [20] studied numerically the problem of mixed convection through a lid-driven air-filled square cavity with a hot wavy wall. Reddy [21] studied the set of non-linear algebraic equation transferred into linear algebraic equations. Zeinkiewicz et al. [22] solved linear equations by applying Triangular Factorization method and reduce integration technique.

The main objective of this work is to present the effect of mixed convection in square lid-driven with internal elliptic body and constant flux heat source on the bottom wall. The detailed analysis of heat transfer rates has been carried out using finite element method. The Richardson number has been varied from 0.01 to 10 by emphasizing dominating forced convection to free convection. Results are presented graphically in terms of streamlines, isotherms, Nusselt number, temperature and velocity profiles.

\section{Physical Configurations}

The physical model considered of a numerical study of mixed convection in square liddriven with internal elliptic body and constant flux heat source on the bottom wall are shown in Fig. 1. The height and the width of the cavity are denoted by $L$ was considered. The left and right wall is kept at cold $T_{c}$ and moving downward. The top wall and internal elliptic block is adiabatic. The bottom wall is adiabatic with heat flux located in the middle.

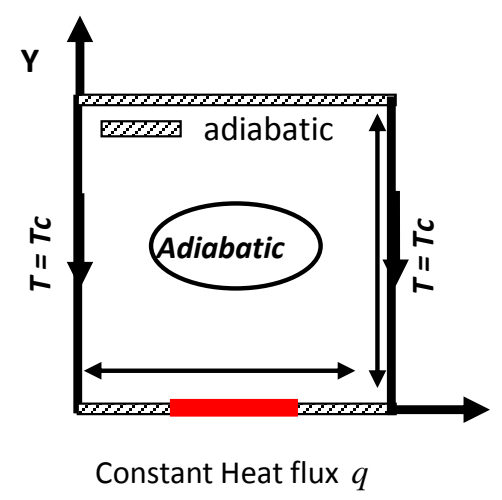

Fig. 1. Schematic view of the cavity with boundary conditions considered in the present paper. 


\section{Mathematical Formulations}

The physical domain is shown in Fig. 1. The hydromagnetic mixed convection flow of enclosed fluid inside the enclosure is assumed to be two-dimensional, steady, laminar, and incompressible. The fluid properties are assumed to be constant except for the density which is considered to vary linearly with temperature according to the Boussinesq approximation. The working fluid is considered to be air $(\operatorname{Pr}=0.733)$. The governing equations for steady mixed convection flow using conservation of mass, momentum and energy can be written as

$$
\begin{aligned}
& \frac{\partial u}{\partial x}+\frac{\partial v}{\partial y}=0 \\
& u \frac{\partial u}{\partial x}+v \frac{\partial u}{\partial y}=-\frac{1}{\rho} \frac{\partial p}{\partial x}+v\left(\frac{\partial^{2} u}{\partial x^{2}}+\frac{\partial^{2} u}{\partial y^{2}}\right) \\
& u \frac{\partial v}{\partial x}+v \frac{\partial v}{\partial y}=-\frac{1}{\rho} \frac{\partial p}{\partial y}+v\left(\frac{\partial^{2} v}{\partial x^{2}}+\frac{\partial^{2} v}{\partial y^{2}}\right)+g \beta\left(T-T_{0}\right) \\
& u \frac{\partial T}{\partial x}+v \frac{\partial T}{\partial y}=\alpha\left(\frac{\partial^{2} T}{\partial x^{2}}+\frac{\partial^{2} T}{\partial y^{2}}\right)
\end{aligned}
$$

The boundary conditions for the present problem are specified as follows:

For upper wall: $u=v=0, \frac{\partial T}{\partial x}=0$

For left and right wall: $u=0, v=-v_{0}, T=T_{c}$

$$
\begin{aligned}
& \text { Bottom wall: } \frac{\partial T}{\partial y}=\left\{\begin{array}{c}
0, \text { for } 0<x<(1-\varepsilon) / 2 \\
-u \text { for }(1-\varepsilon) / 2<x<(1+\varepsilon) / 2 \\
0 \text { for }(1+\varepsilon) / 2<x<1
\end{array}\right. \\
& \text { and } u=v=0
\end{aligned}
$$

where $x$ and $y$ are the distances measured along the horizontal and vertical directions respectively, $u$ and $v$ are the velocity components in $x$ and $y$ direction respectively, $T$ denotes the temperature, $v$ denotes the kinematics viscosity, $\alpha$ denotes the thermal diffusivity respectively, $p$ is the pressure, $\rho$ is the density and $q$ is the uniform constant heat flux respectively.

The equations are non-dimensionalzed by using the following dimensionless quantities

$$
X=\frac{x}{L}, Y=\frac{y}{L}, U=\frac{u}{U_{0}}, V=\frac{v}{U_{0}}, P=\frac{p}{\rho U_{0}^{2}}, \theta=\frac{T-T_{c}}{T_{h}-T_{c}}
$$

where $v=\frac{\mu}{\rho}$ is the reference kinematic viscosity and $\theta$ is the non-dimensional temperature. After substitution of dimensionless variable we get the non- dimensional governing equations:

$$
\begin{aligned}
& \frac{\partial U}{\partial X}+\frac{\partial V}{\partial Y}=0 \\
& U \frac{\partial U}{\partial X}+V \frac{\partial U}{\partial Y}=-\frac{\partial P}{\partial X}+\frac{1}{\operatorname{Re}}\left(\frac{\partial^{2} U}{\partial X^{2}}+\frac{\partial^{2} U}{\partial Y^{2}}\right)
\end{aligned}
$$


$U \frac{\partial V}{\partial X}+V \frac{\partial V}{\partial Y}=-\frac{\partial P}{\partial Y}+\frac{1}{\operatorname{Re}}\left(\frac{\partial^{2} V}{\partial X^{2}}+\frac{\partial^{2} V}{\partial Y^{2}}\right)+R i \theta$

$U \frac{\partial \theta}{\partial X}+V \frac{\partial \theta}{\partial Y}=\frac{1}{\operatorname{Re} \operatorname{Pr}}\left(\frac{\partial^{2} \theta}{\partial X^{2}}+\frac{\partial^{2} \theta}{\partial Y^{2}}\right)$

where $U$ and $V$ are the velocity components in the $X$ and $Y$ directions, respectively, $P$ is the pressure, and $\theta$ is the non-dimensional temperature. Here, all distance are normalized by $W$, all velocities are normalized by $U o$. When $R i \sim 1$ both free and forced convection are equally dominant and the flow regime is designated as mixed convection. If $R i>1$ then free convection is dominant whereas forced convection is dominant when $R i<1$. The boundary conditions for the present problem are specified as follows:

The upper wall: $\frac{\partial \theta}{\partial Y}=0, U=V=0$

$$
\begin{aligned}
& \text { Bottom wall: } \frac{\partial \theta}{\partial Y}=\left\{\begin{array}{c}
0, \text { for } 0<X<(1-\varepsilon) / 2 \\
-1 \text { for }(1-\varepsilon) / 2<X<(1+\varepsilon) / 2 \\
0 \text { for }(1+\varepsilon) / 2<X<1
\end{array}\right. \\
& \text { and } U=V=0
\end{aligned}
$$

Right and left wall: $\theta=0, \mathrm{U}=0, \mathrm{~V}=-1$

We define the local heat transfer coefficient $h_{x}=\frac{q^{n}}{\left[T_{s}-T_{c}\right]}$ at a given point on the heat source surface where $T_{s}(x)$ is the local temperature on the surface. Accordingly the local Nusselt number and the average or over all Nusselt number can be obtained respectively as

$$
\begin{aligned}
& N_{u}=\frac{h_{x} W}{k}=\frac{1}{\theta_{s}(X)} \text { and } \\
& \overline{N u}=\frac{\bar{h} W}{k}=\frac{1}{s} \int_{0}^{E} \frac{1}{\theta_{s}(X)} d X
\end{aligned}
$$

where $\theta_{s}(X)$ is the local dimensionless temperature. The Prandtl number $\operatorname{Pr}=\frac{\gamma}{\alpha}$, the Reynolds number $G r=\frac{g \beta \Delta T W^{\mathrm{s}}}{\gamma^{2}}$, and the Grashof number $G r=\frac{g \beta \Delta T W^{\mathrm{s}}}{\gamma^{2}}$, where $\gamma$ is the kinematic viscosity of the fluid, $\alpha$ is the thermal diffusivity of the fluid, $\beta$ is the thermal expansion coefficient of the fluid, and $g$ is the gravitational acceleration. The ratio $\frac{G r}{R e^{2}}$ is called the Richardson number, $R i$ which represents the relative magnitude of the free convection to the forced convection and plays an important role in designating the convection flow. 


\section{Numerical Techniques}

The nonlinear governing partial differential equations, i.e., mass, momentum and energy equations are transferred into a system of integral equations by using the Galerkin weighted residual finite-element method. The integration involved in each term of these equations is performed with the aid of Gauss quadrature method. The nonlinear algebraic equations so obtained are modified by imposition of boundary conditions. These modified nonlinear equations are transferred into linear algebraic equations with the aid of Newton's method. Lastly, Triangular factorization method is applied for solving those linear equations.

\subsection{Finite element formulation and computational procedure}

The momentum and energy balance equations are the combinations of a mixed ellipticparabolic system of nonlinear partial differential equations. The system of nonlinear partial differential equations (7)-(9) along with boundary conditions are discretized and solve with the aid of the Galerkin weighted residual finite element method.

To derive the finite element equations, the method of weighted residuals in Zienkiewicz [22] is applied to Eqs. (7)-(9) as

$$
\begin{aligned}
& \int_{A} N_{\alpha}\left(U \frac{\partial U}{\partial X}+V \frac{\partial U}{\partial Y}\right) d A=-\int_{A} H_{\lambda}\left(\frac{\partial P}{\partial X}\right) d A+\frac{1}{\operatorname{Re}} \int_{A} N_{\alpha}\left(\frac{\partial^{2} U}{\partial X^{2}}+\frac{\partial^{2} U}{\partial Y^{2}}\right) d A \\
& \int_{A} N_{\alpha}\left(U \frac{\partial V}{\partial X}+V \frac{\partial V}{\partial Y}\right) d A=-\int_{A} H_{\lambda}\left(\frac{\partial P}{\partial Y}\right) d A+\frac{1}{\operatorname{Re}} \int_{A} N_{\alpha}\left(\frac{\partial^{2} V}{\partial X^{2}}+\frac{\partial^{2} V}{\partial Y^{2}}\right) d A+R_{i} \int_{A} N_{\alpha} \theta d A \\
& \int_{A} N_{\alpha}\left(U \frac{\partial \theta}{\partial X}+V \frac{\partial \theta}{\partial Y}\right) d A=\frac{1}{\operatorname{Re} \operatorname{Pr}} \int_{A} N_{\alpha}\left(\frac{\partial^{2} \theta}{\partial X^{2}}+\frac{\partial^{2} \theta}{\partial Y^{2}}\right) d A+\int_{A} N_{\alpha}\left(\frac{\partial^{2} \theta_{s}}{\partial X^{2}}+\frac{\partial^{2} \theta_{s}}{\partial Y^{2}}+Q\right) d A=0
\end{aligned}
$$

Where $A$ is the element area, $N_{\alpha}(\alpha=1,2, \cdots, 6)$ are the element interpolation functions for the velocity components and the temperature, and $H_{\lambda}(\lambda=1,2,3)$ are the element interpolation functions for the pressure.

Gauss's theorem is then applied to Eqs. (10)-(12) to generate the boundary in general terms associated with the surface tractions and heat flux. Then Eqs. (10)-(12) become

$$
\begin{aligned}
& \int_{A} N_{\alpha}\left(U \frac{\partial U}{\partial X}+V \frac{\partial U}{\partial Y}\right) d A+\int_{A} H_{\lambda}\left(\frac{\partial P}{\partial X}\right) d A+\frac{1}{\operatorname{Re}} \int_{A}\left(\frac{\partial N_{\alpha}}{\partial X} \frac{\partial U}{\partial X}+\frac{\partial N_{\alpha}}{\partial Y} \frac{\partial U}{\partial Y}\right) d A=\int_{s_{0}} N_{\alpha} S_{x} d s_{0} \\
& \int_{A} N_{\alpha}\left(U \frac{\partial V}{\partial X}+V \frac{\partial V}{\partial Y}\right) d A+\int_{A} H_{\lambda}\left(\frac{\partial P}{\partial Y}\right) d A+\frac{1}{\operatorname{Re}} \int_{A}\left(\frac{\partial N_{\alpha}}{\partial X} \frac{\partial V}{\partial X}+\frac{\partial N_{\alpha}}{\partial Y} \frac{\partial V}{\partial Y}\right) d A \\
& -R_{i} \int_{A} N_{\alpha} \theta d A=\int_{s_{0}} N_{\alpha} S_{y} d s_{0} \\
& \int_{A} N_{\alpha}\left(U \frac{\partial \theta}{\partial X}+V \frac{\partial \theta}{\partial Y}\right) d A+\frac{1}{\operatorname{RePr}} \int_{A}\left(\frac{\partial N_{\alpha}}{\partial X} \frac{\partial \theta}{\partial X}+\frac{\partial N_{\alpha}}{\partial Y} \frac{\partial \theta}{\partial Y}\right) d A=\int_{S_{w}} N_{\alpha} q_{1 w} d S_{w} \int_{A}\left(\frac{\partial N_{\alpha}}{\partial X} \frac{\partial \theta}{\partial X}+\frac{\partial N_{\alpha}}{\partial Y} \frac{\partial \theta}{\partial Y}\right) d A \\
& =\int_{s w} N_{\alpha} q_{2 w} S_{y} d s_{w}
\end{aligned}
$$


where $S_{x}, S_{y}$ are surface tractions along the outflow boundary $S_{0}$ and $q_{1 w}, q_{2 w}$ are heat flux into or out from the domain along the wall boundary $S_{\mathrm{w}}$ of the element. The basic unknowns for the above differential equations are the velocity components $U$ and $V$, the temperature $\theta$, and the pressure $P$. The five node triangular mesh element is used in this work for the development of the finite element equations. All five nodes are associated with velocities as well as temperature; only the corner nodes are associated with pressure. This means that a lower order polynomial is chosen for pressure, which is satisfied through the continuity equation. Expanding the velocity components $(U, V)$ and temperature $(\theta)$ using the element interpolation function $N_{\alpha}(\alpha=1,2, \cdots, 6)$ and $H_{\lambda}(\lambda=1,2,3)$ as

$U(X, Y)=N_{\beta} U_{\beta}, V(X, Y)=N_{\beta} V_{\beta}, \theta(X, Y)=N_{\beta} \theta_{\beta}, P(X, Y)=H_{\lambda} P_{\lambda}$

Where $\beta=1,2, \ldots, 6$ and $\lambda=1,2,3$

By substituting the element velocity components, temperature and pressure

distributions in Eqs. (13)- (15), the modified finite element equations are

$$
\begin{aligned}
& K_{\alpha \beta \gamma}{ }^{x} U_{\beta} U_{\gamma}+K_{\alpha \beta \gamma} y V_{\gamma} U_{y}+M_{\alpha \mu^{x}} P_{\mu}+\frac{1}{R e}\left(S_{\alpha \beta^{x x}}+S_{\alpha \beta^{y y}}\right) U_{\beta}=Q_{\alpha^{u}} \\
& K_{\alpha \beta Y^{x}} U_{\beta} V_{Y}+K_{\alpha \beta Y^{y}} V_{Y} V_{Y}+M_{\alpha \mu^{x}} P_{\mu}+\frac{1}{R \varepsilon}\left(S_{\alpha \beta^{x x}}+S_{\alpha \beta^{y y}}\right) V_{\beta}- \\
& R i K_{\alpha \beta} \theta_{\beta}=Q_{\alpha^{y}} \\
& K_{\alpha \beta \gamma^{x}} U_{\beta} \theta_{\gamma}+K_{\alpha \beta \gamma} y V_{\beta} \theta_{\gamma}+\frac{1}{R_{\theta} P_{r}}\left(S_{\alpha \beta^{x x}}+S_{\alpha \beta} y y\right) \theta_{\beta}=Q_{\alpha^{\theta}}
\end{aligned}
$$

Where the coefficients in element matrices of the integrals over the element area and along the element edges $S_{0}$ and $S_{w}$ are as

$$
\begin{aligned}
& K_{\alpha \beta^{x}}=\int_{A} N_{\alpha} N_{\beta, x} d A, \quad K_{\alpha \beta^{y}}=\int_{A} N_{\alpha} N_{\beta, y} d A, K_{\alpha \beta \gamma^{x}}=\int_{A} N_{\alpha} N_{\beta} N_{\gamma, x} d A \\
& K_{\alpha \beta^{y}}=\int_{A}^{A} N_{\alpha} N_{\beta} N_{\gamma, y} d A, \quad K_{\alpha \beta}=\int_{A} N_{\alpha} N_{\beta} d A, S_{\alpha \beta^{\mathrm{x}}}=\int_{A} N_{\alpha, x} N_{\beta, x} d A, \\
& S_{\alpha \beta^{y}}=\int_{A} N_{\alpha, y} N_{\beta, y} d A, \quad M_{\alpha \mu^{x}}=\int_{A} H_{\alpha} H_{\mu, x} d A, M_{\alpha \mu^{y}}=\int_{A} H_{\alpha} H_{\mu, y} d A, \\
& Q_{\alpha^{u}}=\int_{S_{0}} N_{\alpha} S_{x} d S_{0}, \quad Q_{\alpha^{y}}=\int_{S_{0}} N_{\alpha} S_{y} d S_{0} \quad M_{\alpha^{\theta}}=\int_{S_{w}} N_{\alpha} q_{1 w} d S_{w}
\end{aligned}
$$

Using Newton-Raphson method of Reddy [21], the set of non-linear algebraic Eqs. (16)- (18) are transferred into linear algebraic equations. Finally, these linear equations are solved by applying Triangular Factorization method and reduced integration technique of Zeinkiewicz et al. [22].

\subsection{Grid refinement check}

We examined five different non-uniform grid systems with the following number of elements within the resolution field. It is observed that grid independence is achieved with 25482 elements where there is insignificant change in $N u$ with further increase of mesh elements. Five different non-uniform grids with the following number of nodes 


\section{A Numerical Study of Mixed Convection}

and elements were considered for the grid refinement tests. From these values, 12991 nodes 25482 elements can be chosen throughout the simulation to optimize the relation between the accuracy required.

Table 1. Grid refinement check at $P r=0.733, R e=10, G r=10$ and $R i=0.01,0.5,1,5,10$.

\begin{tabular}{|l|c|c|c|c|c|}
\hline Nodes & 451 & 616 & 1146 & 3403 & 12991 \\
\hline Elements & 814 & 1127 & 2145 & 6554 & 25482 \\
\hline $\mathrm{Nu}$ & 49.98399 & 49.99788 & 50.01357 & 50.07905 & 50.08104 \\
\hline$\theta_{\alpha v}$ & 0.82835 & 0.81251 & 0.810452 & 0.80167 & 0.80167 \\
\hline
\end{tabular}

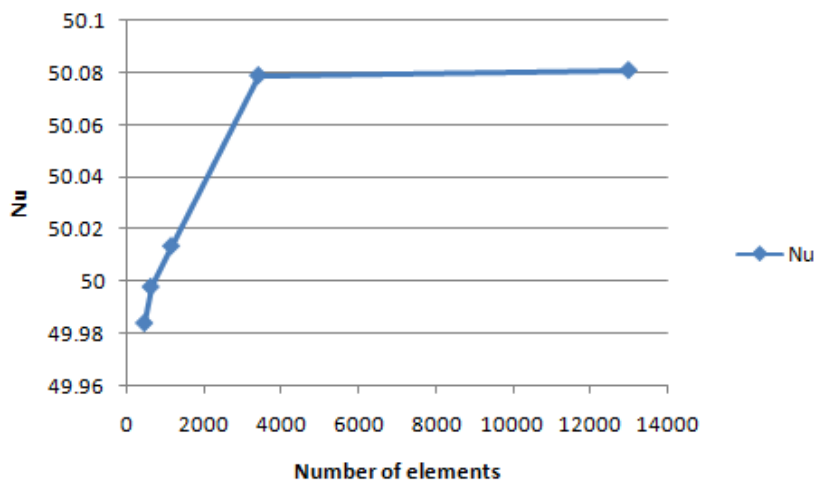

Fig. 2. Grid independency study for different size of elliptic shape when $R i=0.01,0.5,1,5,10$, $\operatorname{Re}=10, G r=10$ and $\operatorname{Pr}=0.733$

\subsection{Mesh generation}

In finite element method, the mesh generation is the technique to subdivide a domain into a set of sub-domains, called finite elements, control volume etc. The discrete locations are defined by the numerical grid, at which the variables are to be calculated. The computational domains with irregular geometries by a collection of finite elements make the method a valuable practical tool for the solution of boundary value problems arising the various fields of engineering. Fig. 3 displays the finite element mesh of the present physical domain.

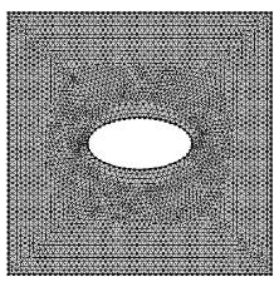

Fig. 3. Mess generation of mixed convection in square lid-driven cavity. 


\section{Program Validations and Comparison with Previous Work}

The computer code and Guanghong et al. [12] was modified and used for the computations in the study. The working fluid is chosen Prandtl number $\operatorname{Pr}=0.733$. The left and right wall is kept at cold $T_{c}$ and moving downward. Both upper and lower are being insulated. By performing simulation for mixed convection in the lower wall is adiabatic. Streamlines and isotherms are plotted in Fig. 4 showing good agreement.

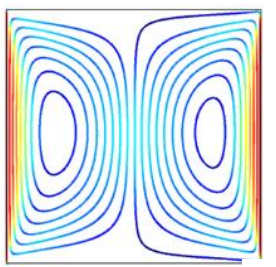

(a)

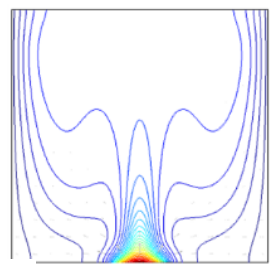

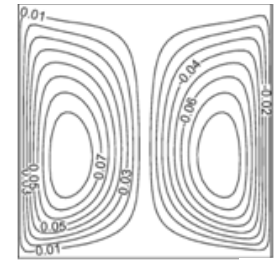

(b)

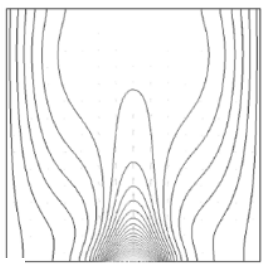

Fig. 4. (a) Present (b) Guanghong et al. obtained streamlines and Isotherms for $R e=10, G r=10$ $P r=0.733$ and $R i=0.01$.

\section{Results and Discussion}

In this section, numerical computations are carried out and a parametric study is performed to illustrate the influence of the physical parameters on the resulting streamlines and isotherms as well as the velocity components at the enclose midsection, Nusselt number, velocity and temperature along the heat sources.
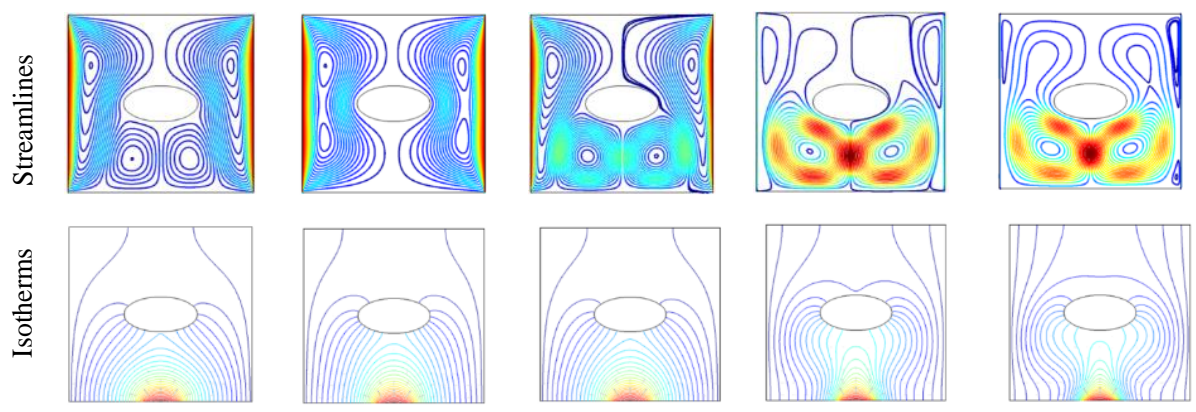

Fig. 5. Stream lines and isotherms for $R i=0.01,0.5,1,5,10, R e=10, G r=10$ and $P r=0.733$.

Fig. 5 illustrates the streamlines and isotherms for different values of Ri. Fig. 5 upper portion shows that the strength of primary and secondary circulations is seen to be very week as the minimum value of circulation is found to be $R i=0.01$. When the Richardson number increases the strength of both primary and secondary circulations increases. The circulation cells are found to be almost symmetric where significant flow is primarily dominated by buoyancy force. The isotherms are found to be smooth 
symmetric curves that span the entire cavity signifying the conduction dominant mode of heat transfer are shown in Fig. 5 lower portion. Fig. 6 shows that the local Nusselt number, vertical velocity, dimensional temperature and temperature gradient. Fig. 6(a), the lower Richardson number, the local Nusselt number has more significant and higher Richardson number the local Nusselt number less significant. Fig. 6(b) variation of the velocity components along the left wall for different $R i$. It can be seen from the figure that the absolute value of maximum and minimum value of the velocity increases with increasing Ri. Fig. 6(c) dimensional temperature line starts at maximum point of left wall and reaches the lower portion to the right wall. Again Fig. 6(d) the variation of temperature starts at maximum points left wall and reaches to the lower wall and moves the upper to the right wall.
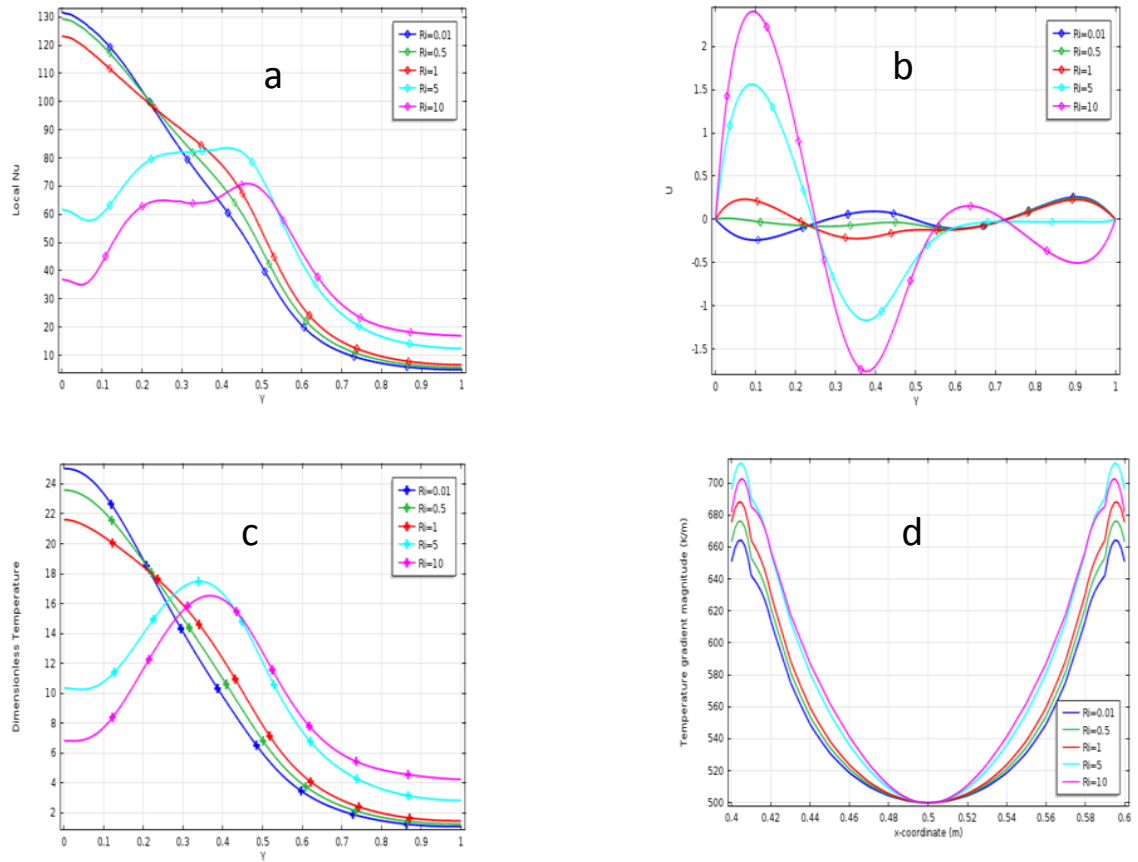

Fig. 6. Local Nussle number, velocity, temperature and temperature gradient for $R i=0.01,0.5$, 1, 5, 10, $R e=10, G r=10$ and $P r=0.733$.

Fig. 7 shows the streamlines and isotherms for mixed convection regime. There are four recirculation eddies which appear when there is no magnetic field presence for $R i=0.01$. Increasing the $R i$ causes the major recirculation eddy at the top wall gradually to become larger and occupy the cavity. From Fig. 7, the variations of Richardson numbers made big impact on the isotherms. At higher $R i$, the isotherms are almost lower walls and satisfied at the upper of the cavity. This indicates that convective flow is weaker and less heat transfer occurs. 
Fig. 8 shows the local Nusselt number, velocity, dimensional temperature and temperature for different Richardson numbers. Fig. 8(a) presents that the local Nusselt number curves started from maximum and minimum points of left wall and fall in the lower part right wall. Fig. 8(b) shows the velocity profile along the horizontal direction at the mid axis in x-direction at different Richardson numbers. Fig. 8(c) shows the dimensionless temperature curve started from upper portion left wall and fall in the lower part right wall. Fig. 8(d) present the temperature started in the upper part the nit is shaply decreased and meet lower wall, next it commenced to increase and finally reached the upper on the right wall. Fig. 9 shows the streamlines and isotherms for mixed convection regime and Fig. 10 shows the Local Nusselt number, velocity, dimensionless temperature and temperature. Both the figure are same as before. The effect of Grashof numbers on the average Nusselt number are presented in Fig. 11. Overall, the average Nusselt number decreases with the increase of Grashof number.
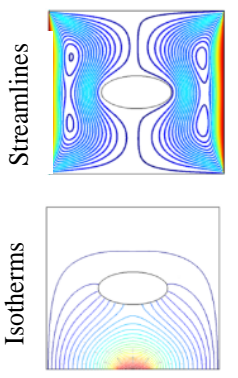
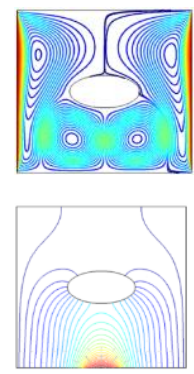
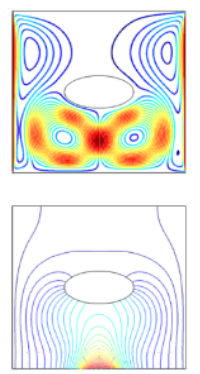
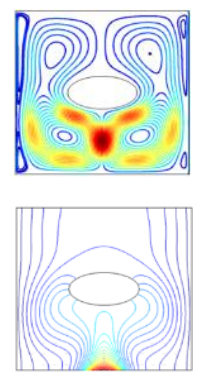
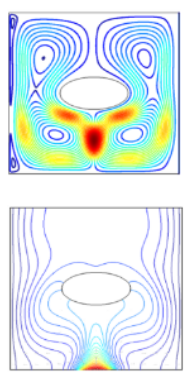

Fig. 7. Stream lines and isotherms for $R i=0.01,0.5,1,5,10, R e=20, G r=20$ and $\operatorname{Pr}=0.733$.
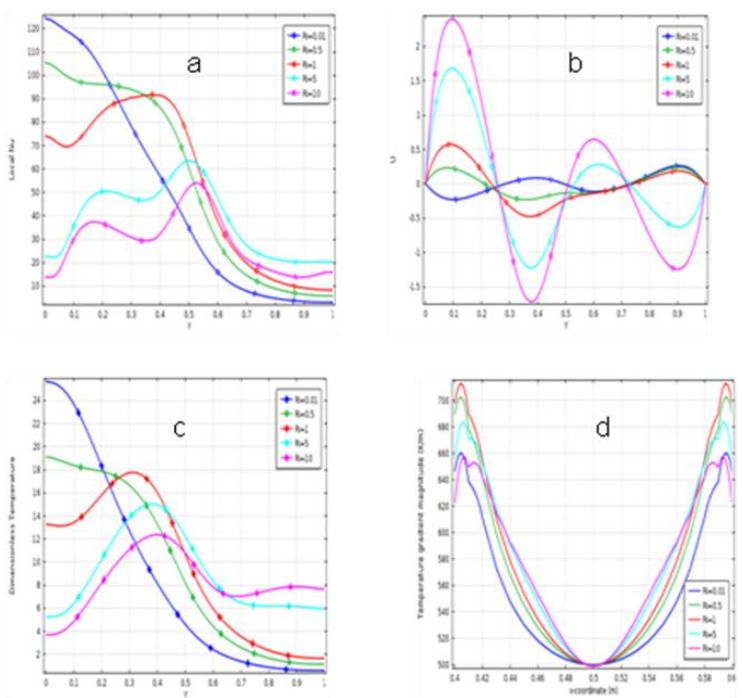

Fig. 8. Local Nussle number, velocity, temperature and temperature gradient for $R i=0.01,0.5$, $1,5,10, R e=20, G r=20$ and $P r=0.733$. 

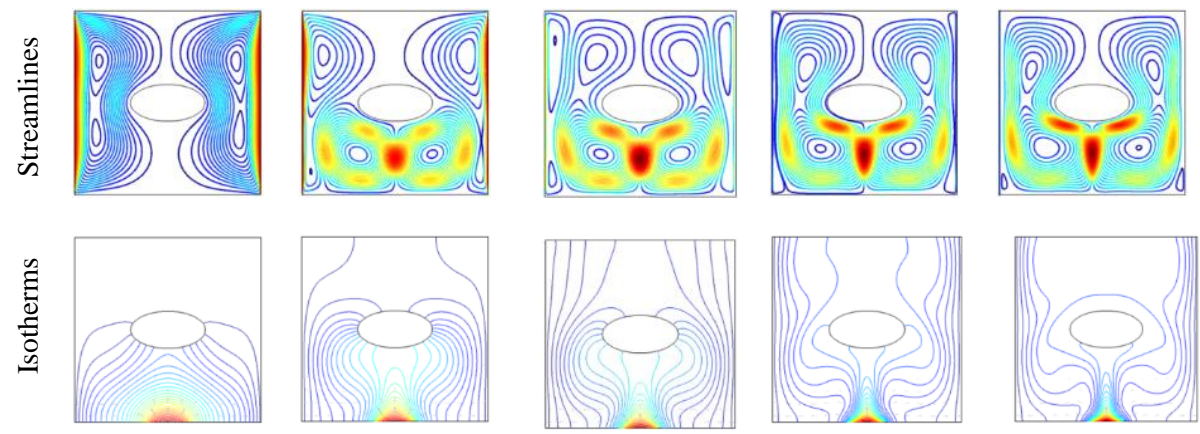

Fig. 9. Stream lines and isotherms for $R i=0.01,0.5,1,5,10, R e=50, G r=50$ and $P r=0.733$.
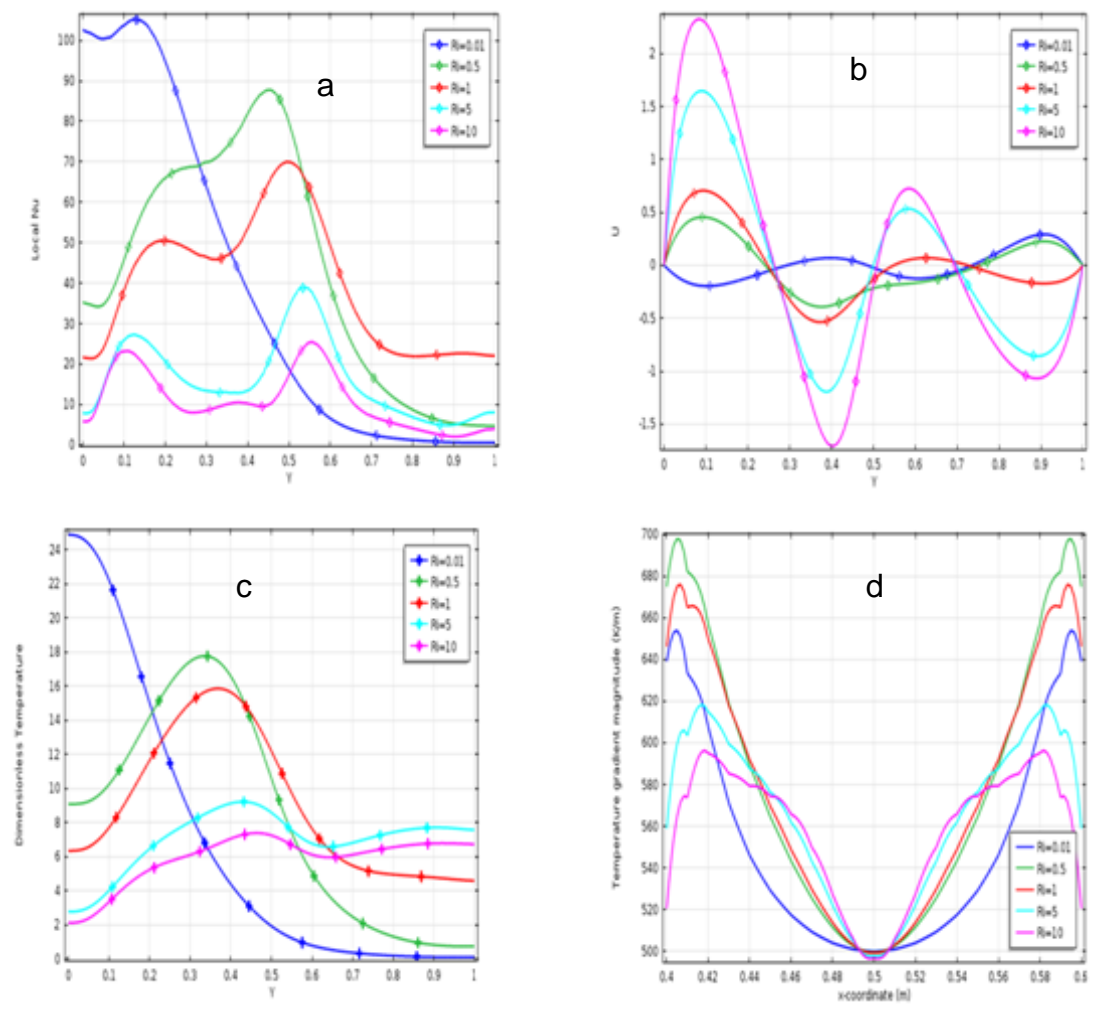

Fig. 10. Local Nussle number, velocity, temperature and temperature gradient for $R i=0.01,0.5$, 1, 5, 10, $R e=20, G r=20$ and $\operatorname{Pr}=0.733$. 


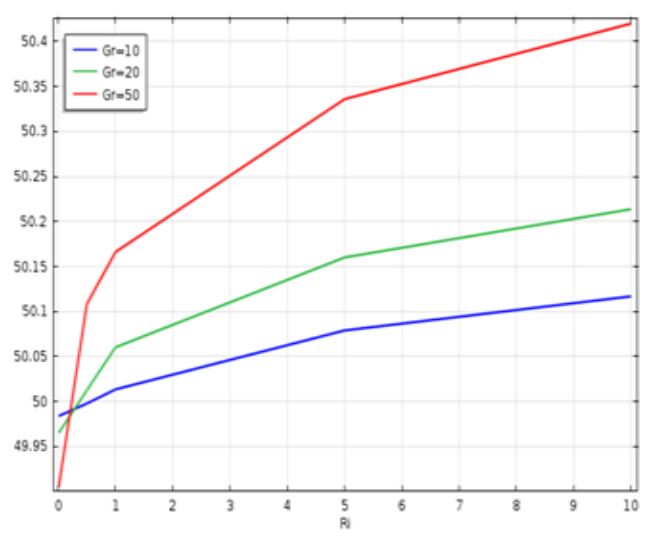

\begin{tabular}{|c|c|c|}
\hline$G r$ & $R i$ & $N u$ \\
\hline \multirow{3}{*}{10} & 0.01 & 49.98399 \\
\cline { 2 - 3 } & 0.5 & 49.99788 \\
\cline { 2 - 3 } & 1 & 50.01357 \\
\cline { 2 - 3 } & 5 & 50.07905 \\
\cline { 2 - 3 } & 10 & 50.11686 \\
\hline \multirow{4}{*}{0} & 0.01 & 49.96525 \\
\cline { 2 - 3 } & 0.5 & 50.01252 \\
\cline { 2 - 3 } & 1 & 50.06032 \\
\cline { 2 - 3 } & 5 & 50.16012 \\
\cline { 2 - 3 } & 10 & 50.21368 \\
\hline \multirow{3}{*}{50} & 0.01 & 49.9054 \\
\cline { 2 - 3 } & 0.5 & 50.10815 \\
\cline { 2 - 3 } & 1 & 50.16609 \\
\cline { 2 - 3 } & 5 & 50.33589 \\
\cline { 2 - 3 } & 10 & 50.41981 \\
\hline
\end{tabular}

Fig. 11. Variation of the average Nusselt numbers and Nusselt numbers table along the bottom wall of the square cavity with Grashof numbers at different Richardson numbers.

\section{Conclusion}

The following conclusions can be drawn from the results of the present work. A mathematical model to simulate mixed convection heat transfer in a two-dimensional square cavity and the associated computer coding has been developed. The model is applied to analyze mixed convection in a square cavity where the cold vertical left and right wall are moving with constant velocity and a constant flux heat source is placed at the bottom. The direction of lid makes important effect on heat transfer. The resulting flow consists of two or more counter-rotating vortices. As far as the temperature field is concerned, at low value of $R i$, when forced convection is the dominant mechanism of heat transfer, the temperature is found to be more evenly distributed within the enclosure, and a relatively large region of the enclosure is affected by the heat source. As $R i$ increases the temperature variation is restricted over a gradually diminishing regime around the heat source. It is also noticed that the heataffected region becomes larger with the increasing heat source length. Higher heat transfer is observed for the case of downward moving wall.

\section{Appendix: Nomenclature}

\begin{tabular}{|c|c|c|c|}
\hline$g$ & gravitational acceleration & $X, Y$ & $\begin{array}{l}\text { Non-dimensional Cartesian } \\
\text { coordinates }\end{array}$ \\
\hline$L$ & length of the cavity & FEM & Finite Element Method \\
\hline$N u$ & Nusselt number & \multicolumn{2}{|c|}{ Greek symbols } \\
\hline $\boldsymbol{P}$ & dimensional pressure & $\alpha$ & thermal diffusivity \\
\hline$p$ & pressure & $\boldsymbol{\beta}$ & $\begin{array}{l}\text { Volumetric coefficient of } \\
\text { thermal expansion }\end{array}$ \\
\hline $\operatorname{Pr}$ & Prandtl number & $v$ & kinematic viscosity of the fluid \\
\hline$R \boldsymbol{a}$ & Rayleigh number & $\boldsymbol{\theta}$ & non-dimensional temperature \\
\hline $\operatorname{Re}$ & Reynolds number & $\rho$ & density of the fluid \\
\hline$R i$ & Richardson number & $\psi$ & $\begin{array}{l}\text { non-dimensional stream } \\
\text { function }\end{array}$ \\
\hline
\end{tabular}




\begin{tabular}{llll}
$\boldsymbol{G r}$ & Grashof number & \multicolumn{2}{c}{ Subscripts } \\
$\boldsymbol{T}$ & dimensional temperature & $\boldsymbol{c}$ & cold wall \\
$\boldsymbol{u}, \boldsymbol{v}$ & velocity components & $\boldsymbol{h}$ & hot wall \\
$\boldsymbol{U}, \boldsymbol{V}$ & non-dimensional velocity & & \\
& components & & \\
$\boldsymbol{x}, \boldsymbol{y}$ & Cartesian coordinates & &
\end{tabular}

\section{Acknowledgments}

This research would not be possible without the support of Department of Mathematics, Bangladesh University of Engineering and Technology (BUET).

\section{References}

1. F. P. Incropera, Convection heat transfer in electronic equipment cooling, J. Heat Transfer 110 (1988) 1097-1111.

2. A. M. Al-Amiri, K. M. Khanafer, and I. Pop, Int. J. Thermal Sci. 46, 662 (2007). https://doi.org/10.1016/j.ijthermalsci.2006.10.003

3. M. A. R. Sharif, Appl. Thermal Eng. 27, 1036 (2007). https://doi.org/10.1016/j.applthermaleng.2006.07.035

4. K. M. Khanafer, A. M. Al-Amiri, and I. Pop, Eur. J. Mechanics B/Fluids 26, 669 (2007). https://doi.org/10.1016/j.euromechflu.2006.06.006

5. M. M. Rahman, M. A. Alim, and M. M. A. Sarker, Int. Commun. Heat Mass Transfer 37, 524 (2010). https://doi.org/10.1016/j.icheatmasstransfer.2009.12.012

6. L. K. Saha, M. C. Somadder, and K. M. S. Uddin, Am. J. Appl. Math. 1(5), 92 (2013). https://doi.org/10.11648/j.ajam.20130105.11

7. M. M. Rahman, M. A. Alim and M. A. H. Mamun, Nonlinear Anal. :Model. Control 14, 217 (2009).

8. L. K. Saha, K. M. S. Uddin, and M. A. Taher, Am. J. Appl. Math. 3, 20 (2015).

9. L. K. Saha and M. C. Somadder, Am. J. Appl. Math. 3, 8 (2015). https://doi.org/10.11648/j.ajam.20150301.13

10. G. Guo and M. A. R. Sharif, Int. J. Thermal Sci. 43465 (2004). https://doi.org/10.1016/j.ijthermalsci.2003.08.008

11. N. A. Hussein, J. Babylon University/Eng. Sci. 21(2), 616 (2013).

12. M. J. H. Munshi, M. A. Alim, and G. Mostafa, Int. J. Eng. Appl. Sci. 3(6), 42 (2016).

13. R. Nasrin, J. Sci. Res. 3(3), 501 (2011). https://doi.org/10.3329/jsr.v3i3.7433

14. S. Hussain and A. Hussein, Int. Commun. Heat Mass Transfer 38, 263 (2011). https://doi.org/10.1016/j.icheatmasstransfer.2010.12.006

15. S. Sivasankaran, V. Sivakumar, and A. Hussein, Int. Commun. Heat Mass Transfer 46, 112 2013. https://doi.org/10.1016/j.icheatmasstransfer.2013.05.022

16. S. Sivasankaran, V. Sivakumar, A. Hussein, and P. Prakash, Num. Heat Transfer- Part A, 65, 269 (2014). https://doi.org/10.1080/10407782.2013.826017

17. A. Hussein, J. Basic Appl. Scientific Res. 3(10), 328-338 (2013).

18. A. Hussein, and S. Hussain, J. Thermal Eng. 1(3), 221 (2015). https://doi.org/10.18186/jte.66113

19. S. Saha, A. Hussein, G. Saha, and S. Hussain, Int. J. Heat Technol. 29(1), 143 (2011).

20. A. Hussein, and S. Hussain, Int. J. Mech. Mater. Eng. 5(2), 222 (2010).

21. J. N. Ready, An Introduction to Finite Element Analysis (McGraw-Hill, New York, 1993).

22. O. C. Zeinkiewicz, R. L. Taylor, and J. M. Too, Int. J. Num. Methods Eng. 3, 275 (1971). https://doi.org/10.1002/nme.1620030211 